\title{
Engineering molecular chains in carbon nanotubes $\dagger$
}

\author{
Thomas W. Chamberlain, ${ }^{a}$ Rudolf Pfeiffer, ${ }^{b}$ Jonathan Howells, ${ }^{a}$ Herwig Peterlik, ${ }^{* b}$ Hans Kuzmany, ${ }^{b}$ \\ Bernhard Kräutler, ${ }^{c}$ Tatiana Da Ros, ${ }^{d}$ Manuel Melle-Franco, ${ }^{e}$ Francesco Zerbetto ${ }^{f}{ }$ Dragana Milić ${ }^{g}$ \\ and Andrei N. Khlobystov*a
}

\author{
Received 3rd September 2012, Accepted 20th September 2012 \\ DOI: 10.1039/c2nr32571c
}

\begin{abstract}
A range of mono- and bis-functionalised fullerenes have been synthesised and inserted into singlewalled carbon nanotubes. The effect of the size and shape of the functional groups of the fullerenes on the resultant 1D arrays formed within the nanotubes was investigated by high resolution transmission electron microscopy and X-ray diffraction. The addition of non-planar, sterically bulky chains to the fullerene cage results in highly ordered 1D structures in which the fullerenes are evenly spaced along the internal nanotube cavity. Theoretical calculations reveal that the functional groups interact with neighbouring fullerene cages to space the fullerenes evenly within the confines of the nanotube. The addition of two functional groups to opposite sides of the fullerene cages results in a further increase in the separation of the fullerene cages within the nanotubes at the cost of lower nanotube filling rates.
\end{abstract}

\section{Introduction}

Filling single-walled carbon nanotubes (SWNTs) has become a hot topic in the field of nanomaterial science over recent years. The insertion of molecules and ions into SWNTs is one of the most powerful methods available for organising matter into quasi-1D chains at the nanoscopic level. The spatial confinement imposed by the nanotube can result in dramatic changes in the structural and dynamic properties of the guest species, facilitating the formation of otherwise unobtainable packing motifs ${ }^{1}$ and significantly altering both molecular translation ${ }^{2,3}$ and rotation. ${ }^{3}$ The interior of the nanotube can also be utilised to alter the reactivity of the encapsulated molecules, ${ }^{4}$ and as a template, leading to the formation of new nanostructures possessing exciting properties. ${ }^{5}$

By far the most common species inserted into carbon nanotubes is fullerenes, where the commensurate match of the internal wall of the nanotube (concave) and the spherical fullerenes

${ }^{a}$ Department of Chemistry, University of Nottingham, Nottingham, UK E-mail: andrei.khlobystov@nottingham.ac.uk; Fax: +44 (0)115951 3563; Tel: +44 (0)1159513917

${ }^{b}$ Faculty of Physics, University of Vienna, Austria. E-mail: herwig. peterlik@univie.ac.at; Fax: +4314277 9729; Tel: +431427751350

'Institute of Organic Chemistry, University of Innsbruck, Austria

${ }^{d}$ Department of Chemical and Pharmaceutical Sciences, University of Trieste, Italy

${ }^{e}$ CCTC, Department of Informatics, University of Minho, 4710-057, Braga, Portugal

fDipartimento di Chimica "G. Ciamician", Università di Bologna, V. F. Selmi 2, 40126 Bologna, Italy

${ }^{g}$ Faculty of Chemistry, University of Belgrade, Serbia

$\dagger$ Electronic supplementary information (ESI) available: HRTEM images of 4@SWNT, space filling models of 1-6@SWNT structures and crystal packing considerations of 6 . See DOI: 10.1039/c2nr32571c (convex) results in efficient van der Waals interactions (up to $288 \mathrm{~kJ} \mathrm{~mol}^{-1}$ for $\left.\mathrm{C}_{60}\right)^{6}$ which act as the driving force for the irreversible encapsulation of the fullerene molecules. The rich and varied reactivity of fullerenes has allowed a wide variety of functional groups to be chemically bound to the carbon cage and the resultant functionalised fullerenes inserted into SWNTs. This methodology has been utilised to introduce a host of organic functional groups, ${ }^{5,7-9}$ transition metal centres, ${ }^{10-12}$ and electron spin-active groups ${ }^{13-17}$ into carbon nanotubes.

Such encapsulated molecules can be regarded as dopants, which can locally perturb and modulate the intrinsic properties of the nanotubes. ${ }^{18-22}$ This offers a methodology for tuning the functional properties of nanotubes, such as the electronic band gap, and the concentration and mobility of charge carriers. ${ }^{23-25}$

The utilisation of nanotubes as containers for magnetic guestmolecules is particularly important for several applications such as spintronics and quantum information processing. As spin active molecules can be viewed as potential carriers of quantum information, ${ }^{22}$ the ability to control the spin concentration and separation between the spins in carbon nanotubes is crucial to the successful application of such nanostructures. Previous attempts to engineer the density of magnetic guest-species within SWNTs have involved the insertion of a mixture of spin-carrying endohedral and spin-silent empty fullerene cages, so that the ratio of these molecules defines the average distance between spin-carrying molecules. ${ }^{15,17,26}$ Due to the random nature of the nanotube filling process, the local structure in such molecular chains will be disordered with the distance between neighbouring spin carriers varying statistically from them being located next to each other to a situation in which they are separated by numerous spin-silent species. An alternative method of controlling the intermolecular separation is to use fullerene molecules 
that have been chemically modified with bulky functional groups which, once encapsulated within the confines of the nanotube, will provide a steric barrier between adjacent cages and can be utilised to controllably increase the cage-to-cage separation of the arrays of fullerenes and hence precisely control the number of molecules per unit length of nanotube, Fig. 1.

This strategy was first tested for fullerenes functionalised with alkyl chains of different lengths. ${ }^{27}$ These long, flexible chains can potentially give large interfullerene spacings, measured experimentally as the distance between the centres of two adjacent fullerene cages within the nanotube (centre-to-centre distance), however, the flexibility of the alkyl chain, which enables the spacer to fold up unpredictably, led to a wide variety of intermolecular separations and loss of periodicity, Fig. 1b. A second model used for this approach was based on fullerenes functionalised with rigid, planar aromatic aryl-groups which, in contrast to the alkyl chains, could only adopt a very limited number of conformations. ${ }^{28}$ However, the aromatic groups were found to be able to slip between the neighbouring fullerene cages and the nanotube walls (Fig. 1c). The resultant molecular arrays of functionalised fullerenes are more uniform but the interfullerene distances are significantly reduced in these structures (Table 1).

An additional consideration is that, although all previous attempts to control inter-fullerene separations have involved the use of mono-functionalised fullerenes, this strategy disregards the stochastic nature of the process of the individual fullerene molecules entering the SWNTs, thus making it impossible to control whether a fullerene enters the nanotube cage (head) first or side-group (tail) first. In general, both of these cases are possible, which will result in three possible orientations of two adjacent fullerenes (Fig. 1a). This increases the variation in the

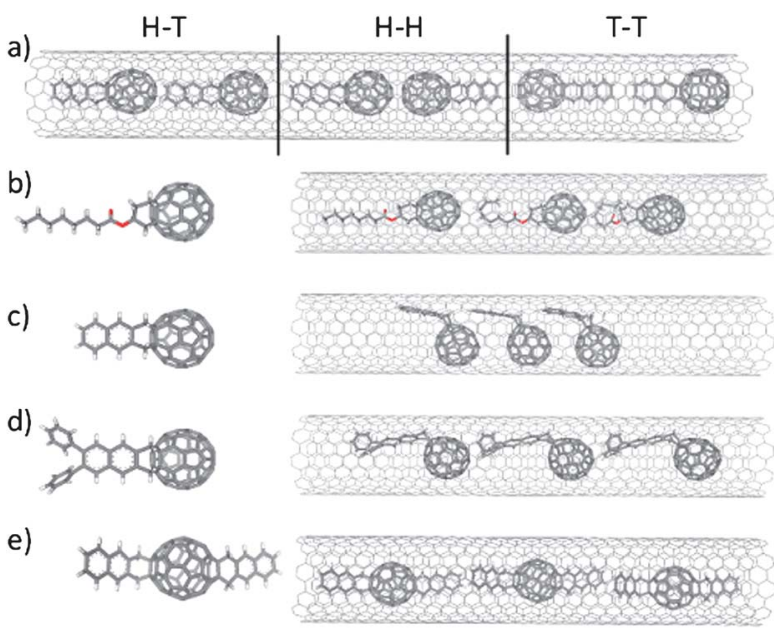

Fig. 1 Schematic diagrams of functionalised fullerenes inside SWNTs depicting: (a) head-to-tail $(\mathrm{H}-\mathrm{T})$, head-to-head $(\mathrm{H}-\mathrm{H})$, and tail-to-tail (T-T) configurations of mono-functionalised fullerenes, (b) reduced uniformity of a fullerene array due to buckling of the flexible functional groups, (c) mono-functionalised cages with planar side-groups that slip between the neighbouring cage and the nanotube sidewall, (d) fullerenes with rigid functional groups with bulky ends to prevent slipping similar to (c), and (e) bis-functionalised fullerenes which allow only one possible interfullerene orientation ( $\mathrm{T}-\mathrm{T})$ providing improved ordering in the molecular arrays.
Table 1 Mean interfullerene spacings for pristine $\mathrm{C}_{60}$ and alkyl-27 and aryl-functionalised ${ }^{28}$ fullerenes encapsulated in nanotubes

\begin{tabular}{lll}
\hline Type of structure & $\begin{array}{l}\text { Mean interfullerene } \\
\text { separation }(\mathrm{nm})\end{array}$ & $\begin{array}{l}\text { Increase in separation } \\
\text { from } \mathrm{C}_{60} @ \text { SWNT }(\mathrm{nm})\end{array}$ \\
\hline $\mathrm{C}_{60} @$ SWNT & $0.98 \pm 0.01$ & - \\
Alkyl fullerenes & & 0.31 \\
$\mathrm{C}_{60}\left(\mathrm{C}_{14} \mathrm{H}_{27} \mathrm{ON}\right) @$ SWNT & $1.29 \pm 0.65$ & 0.85 \\
$\mathrm{C}_{60}\left(\mathrm{C}_{23} \mathrm{H}_{44} \mathrm{O}_{2}\right) @$ SWNT & $1.83 \pm 0.29$ & \\
Aryl fullerenes & & 0.17 \\
$\mathrm{C}_{60}\left(\mathrm{C}_{14} \mathrm{H}_{10} \mathrm{~N}_{2}\right) @$ SWNT & $1.15 \pm 0.12$ & 0.19 \\
$\mathrm{C}_{60}\left(\mathrm{C}_{18} \mathrm{H}_{12} \mathrm{~N}_{2}\right) @$ SWNT & $1.17 \pm 0.10$ & \\
\hline
\end{tabular}

observed interfullerene distances and reduces the uniformity of the generated $1 \mathrm{D}$ arrays.

In this study we utilise functionalised fullerenes specifically designed to overcome all of the above problems and improve the precision of the construction of molecular arrays inside nanotubes.

Three mono-functionalised fullerenes, which contain rigid aromatic side-groups with non-planar, bulky end-groups, along with three bis-functionalised fullerenes were synthesised and their behaviour in nanotubes was explored. The rigid nature of the attached side-groups and the non-planar end groups is designed to prevent the close packing of fullerene cages due to slipping of the functional groups between neighbouring fullerenes and the nanotube sidewall (Fig. 1d). Each of the three bisfunctionalised molecules contains two functional groups located on opposite sides of the fullerene cage, which upon encapsulation should form a single packing motif resulting in highly periodic structures with uniform cage-to-cage spacing possible (Fig. 1e).

\section{Results and discussion}

In this study three mono-functionalised fullerenes, 7-tert-butyl$1^{\prime}, 2^{\prime}, 3^{\prime}, 4^{\prime}$-tetrahydrophenazine-[1,9] $\left(\mathrm{C}_{60}-I_{\mathrm{h}}\right)[5,6]$ fullerene, $\mathbf{1}$, $6^{\prime}, 7^{\prime}, 8^{\prime}, 9^{\prime}$-tetrahydro-2,3-di(pyridin-2-yl)benzoquinoxaline-[1,9]$\left(\mathrm{C}_{60}-I_{\mathrm{h}}\right)[5,6]$ fullerene, 2 , and 9,10-dihydroanthracene-[1,9]- $\left(\mathrm{C}_{60^{-}}\right.$ $\left.I_{\mathrm{h}}\right)[5,6]$ fullerene (the mono-adduct of anthracene and $\left.\mathrm{C}_{60}\right), \mathbf{3}$, and three bisfunctionalised fullerenes; di((4-tert-butylphenyl)cyclohex-2-enone)-[1,9;55,60] $\left(\mathrm{C}_{60}-I_{\mathrm{h}}\right)[5,6]$ fullerene, $\mathbf{4}, 2^{\prime} H, 2^{\prime \prime} H$-[5,6] fullereno- $\mathrm{C}_{60}-I_{\mathrm{h}}-\left[1,9-c: 49,59-c^{\prime}\right]$ dipyrrole- $1^{\prime}\left(5^{\prime} H\right)$-propanoic acid, $\quad 1^{\prime \prime}-[2-[[(9 H$-fluoren-9-ylmethoxy)carbonyl $]$ amino $]$ ethyl $]-$ $1^{\prime \prime}, 5^{\prime \prime}$-dihydro-,1,1-dimethylethyl ester, 5, and di-9',10'-dihydroanthraceno- $[9,10 ; 55,60]\left(\mathrm{C}_{60}-I_{\mathrm{h}}\right)[5,6]$ fullerene (the transpodal bis-adduct of anthracene and $\mathrm{C}_{60}$ ), 6, were synthesised (Fig. 2).

The synthesis of $\mathbf{1}$ and $\mathbf{2}$ was performed using an adapted synthetic route based on the work by Mattay et al. ${ }^{29}$ The synthesis of 3 and 6 has been reported previously. ${ }^{30-33}$ The bisfunctionalised 4 was prepared by a multi-step synthesis. ${ }^{34} \mathbf{5}$ was prepared by first synthesising the (tert-butyl propionate)pyrrolidine[60]fullerene and then allowing it to react with $N-(2-\{[(9-$ fluorenylmethoxy)carbonyl]amino ethyl)glycine in the presence of paraformaldehyde. ${ }^{35}$ The bisadducts were separated by flash chromatography to obtain the trans-2 isomer.

Two of the fullerenes contain rigid aromatic side-groups with non-planar, bulky ends, $\mathbf{1}$ and $\mathbf{2}$, and one has a covalently linked dihydroanthracene moiety providing significant three-dimensional steric bulk, 3. All molecules have very limited 


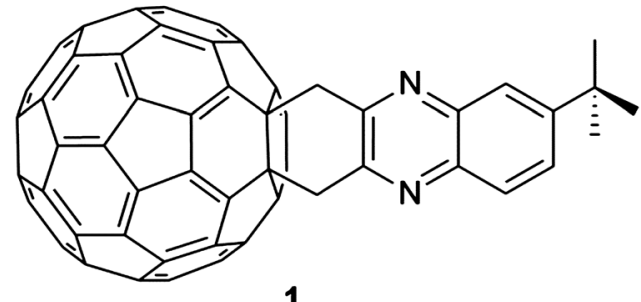

1

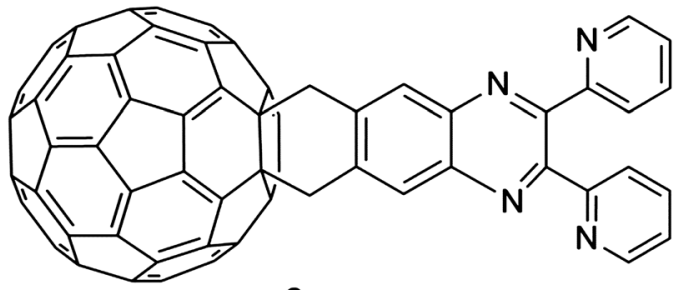

2

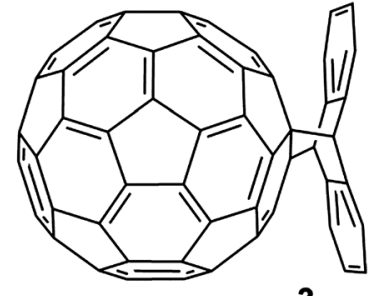

3

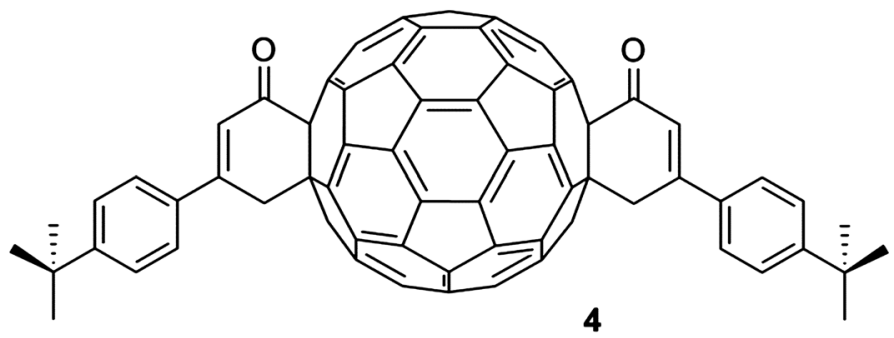

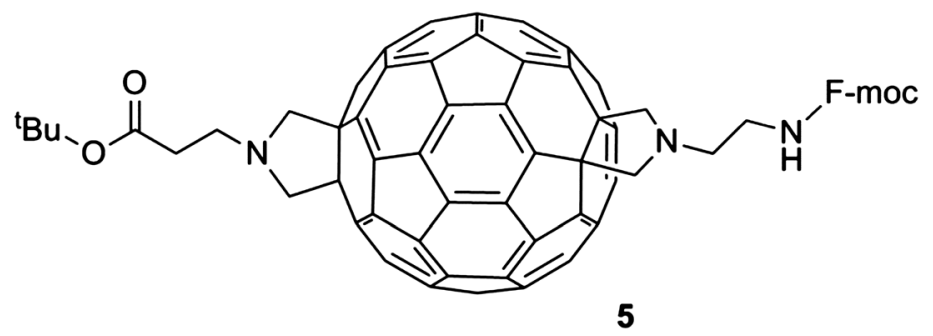

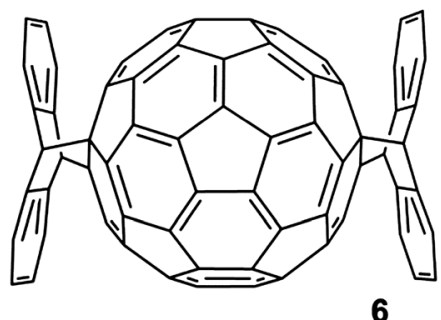

Fig. 2 Structural diagrams of functionalised fullerenes, 1-6.

conformational flexibility which reduces the variation of packing arrangements inside the nanotube, hence leading to periodically spaced arrays. The incorporation of bulky, non-planar functionality at the end of the side-groups should also lead to more regular arrays due to the reduced number of ways in which the groups can be accommodated within the limited space inside the nanotubes. The two functional groups of each bis-functionalised fullerene are located on opposite sides of the carbon cage. The relative position of the two functional groups on the $\mathrm{C}_{60}$ cage is important as any other isomers of $\mathbf{4}$ and $\mathbf{6}$ would not be able to enter the narrow channel of the SWNT (typical $d_{\mathrm{NT}}=1.2-$ $1.6 \mathrm{~nm})$. Upon encapsulation the bis-functionalised fullerenes should form a single packing motif resulting in a regular structure with only one cage-to-cage spacing possible (T-T). Comparison of the molecular arrays formed by fullerenes 3 and 6, the mono-adduct and trans-podal bis-adduct of anthracene and $\mathrm{C}_{60}$ respectively, will enable the effect of mono- and bisfunctionalisation of $\mathrm{C}_{60}$ using the same side-group to be quantified.

For pristine $\mathrm{C}_{60}$ (and other molecules), the most effective method of insertion into a SWNT is from the gas phase. ${ }^{36}$ The opened nanotubes are placed with an excess of fullerene powder inside an evacuated quartz tube and heated to a temperature above the sublimation point of the fullerene. The gas phase fullerene molecules enter the SWNTs and become irreversibly encapsulated, resulting in an extremely high ratio of filled nanotubes (up to $100 \%$ ). However, for functionalised fullerenes, which decompose at elevated temperatures rather than sublime, this route is not viable. ${ }^{3}$ Therefore, a solution-based method has been developed in which functionalised fullerene molecules can be inserted into SWNTs at room temperature which ensures that the thermally sensitive molecules remain intact during the encapsulation process. ${ }^{11}$ Fullerenes 1-6 were inserted into nanotubes by suspending freshly opened SWNTs in a saturated suspension of the respective fullerene species in chloroform followed by evaporation of the solvent, which enables the molecules to irreversibly enter the nanotube interior. ${ }^{11}$ Extensive washing with carbon disulphide removes any fullerene material from the outside of the nanotube and ensures that any fullerene molecules retained in the sample are located within the nanotubes. ${ }^{27} \mathrm{~A}$ sample of $\mathrm{C}_{60} @$ @SWNT was also prepared for comparison using the same conditions.

The resultant structures were characterised by X-ray diffraction (XRD) and high resolution transmission electron microscopy (HRTEM) at $100 \mathrm{kV}$ in which the fullerene cage can be clearly observed as a circle within the confines of the nanotube (seen as two parallel lines in Fig. 3). HRTEM demonstrates filling ratios for all mono-functionalised fullerenes, 1, 2 and 3, comparable to those previously observed for functionalised fullerenes (Table 2). ${ }^{27,28}$ Arrays of fullerenes (>20 molecules) which extend beyond the field of view of the TEM image are observed for all samples. As in previous studies, ${ }^{27,28}$ it was not possible to resolve the functional groups but the elongated shape of the fullerene cage and the increased interfullerene spacing were taken as conformation of functionalised fullerene encapsulation. Quantitative analysis of the interfullerene distances for the 


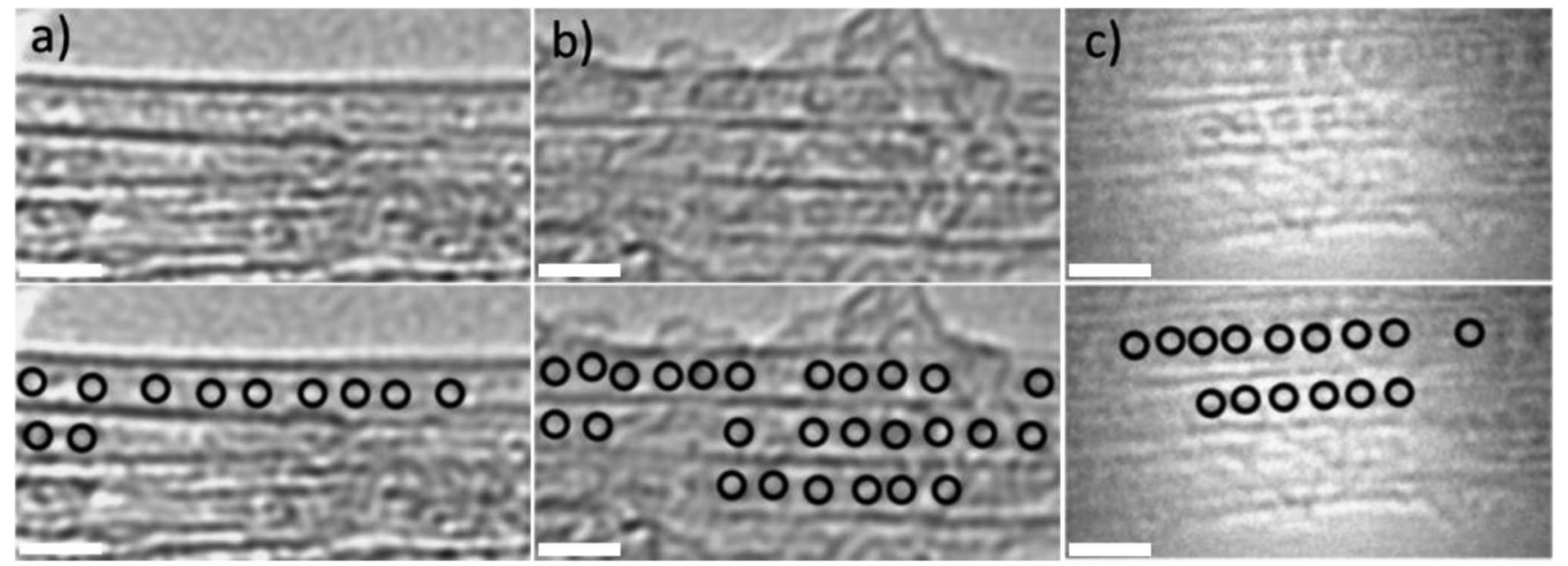

Fig. 3 HRTEM images of (a) 1@SWNT, (b) 2@SWNT, and (c) 3@SWNT. Scale bar $2 \mathrm{~nm}$. In the lower images, the fullerene positions are highlighted by circles.

mono-functionalised fullerenes (Fig. 6 and Table 2) indicates that all functionalised fullerenes show an increase in separation compared to $\mathrm{C}_{60} @ \mathrm{SWNT}$ which is proportional to the size of the functional group (Table 2).

In comparison to the mono-functionalised, alkylated fullerenes studied previously (Table 1) we observe similar-sized interfullerene spacings for 1@SWNT and 2@SWNT but with much improved uniformity, as indicated by the significantly smaller standard deviations in the measured separations $(0.06$ and $0.05 \mathrm{~nm}$ vs. 0.65 and $0.29 \mathrm{~nm}$ for fullerenes 1 and 2 and the previously reported alkyl fullerenes respectively). In fact, the uniformity of the intermolecular spacing for $\mathbf{1}$ and $\mathbf{2}$ is better than that of the previously reported aryl-functionalised fullerenes containing rigid groups. ${ }^{28} 3 @$ SWNT demonstrates a similar wellordered structure. Non-planar, sterically bulky end groups, introduced in 1-3, successfully prevent any slipping of the functional groups past neighbouring molecules, which results in larger and much more regular spacings in their molecular arrays.

The filling ratios for all three bis-functionalised fullerenes, 4-6, were dramatically lower than for the mono-functionalised fullerenes. However, sufficient numbers of molecules were found in nanotubes for samples 4@SWNT and 6@SWNT to allow statistical analysis to be conducted (Table 2). The slightly angular shape of $\mathbf{5}$, resulting from the two functional groups not being located directly opposite each other on the fullerene cage, and possible non-covalent interactions between the functional groups of 5 and SWNT exteriors appear to prevent the molecules from entering the nanotube.

As expected from the respective sizes of the functional groups, 4@SWNT exhibits the largest spacing between the adjacent fullerene cages observed so far for all functionalised fullerenes (1.98 nm, ESI file $\dagger$ and Table 2). Additionally, molecules of 4 appear to be well ordered (standard deviation $=0.14 \mathrm{~nm}$ ), which compares favourably with the values previously reported for mono-functionalised fullerenes (standard deviations $=0.12$ $0.65 \mathrm{~nm}$ ) (Table 1). For 6@SWNT the measured spacing was $1.19 \pm 0.16 \mathrm{~nm}$ (Fig. 4 and Table 2) which is in the same range as for the aryl functionalised fullerenes and is only slightly larger than for the mono-anthracene adduct, 3@SWNT.

The interfullerene separation distances for fullerenes 1-4 and $\mathbf{6}$ encapsulated in a $(10,10) \operatorname{SWNT}\left(d_{\mathrm{SWNT}}=1.39 \mathrm{~nm}\right)$ were estimated using a simple space filling model in which the geometrically optimised functionalised fullerenes are arranged in a $(10,10)$ SWNT so that they are packed in close contact with neighbouring fullerene molecules within the confines of the nanotube sidewalls. The resultant interfullerene spacings for pairs of functionalised fullerene molecules were calculated for all three potential packing arrangements (ESI file $\dagger$ ) and are summarised

Table 2 Filling ratios and fullerene spacings for molecular arrays of 1-6 in SWNT (a sample of $\mathrm{C}_{60} @$ SWNT was prepared under the same conditions for comparison): filling ratios were determined by HRTEM; expected interfullerene spacings as calculated (ESI file $\dagger$ ); interfullerene spacings as measured by HRTEM; interfullerene spacings as measured by XRD (s indicates a strong peak and w a weak peak); effective increase in interfullerene spacing vs. $\mathrm{C}_{60} @$ SWNT from HRTEM

\begin{tabular}{llllll}
\hline Type of structure & Filling ratio/\% & $\begin{array}{l}\text { Expected interfullerene } \\
\text { spacing (H-T orientation)/nm }\end{array}$ & $\begin{array}{l}\text { HRTEM interfullerene } \\
\text { spacing/nm }\end{array}$ & $\begin{array}{l}\text { XRD interfullerene } \\
\text { spacing/nm }\end{array}$ & $\begin{array}{l}\text { HRTEM increase } \\
\text { in spacing/nm }\end{array}$ \\
\hline C60@SWNT & $60-65$ & 1.0 & $0.98 \pm 0.01$ & $-1.55 \mathrm{~s} ; 1.13 \mathrm{w}$ & - \\
1@SWNT & $15-20$ & 1.9 & $1.41 \pm 0.06$ & $1.59 \mathrm{~s} ; 1.12 \mathrm{w}$ & 0.43 \\
2@SWNT & $10-15$ & 2.0 & $1.46 \pm 0.05$ & $1.45 ; 1.16$ & 0.16 \\
3@SWNT & $20-25$ & 1.2 & $1.14 \pm 0.14$ & 2.67 & 1.00 \\
4@SWNT & $2-3$ & 2.6 & N/A & N/A & 0.21 \\
5@SWNT & 0 & - & $1.19 \pm 0.16$ & 3.45 & \\
6@SWNT & $10-15$ & 1.6 &
\end{tabular}




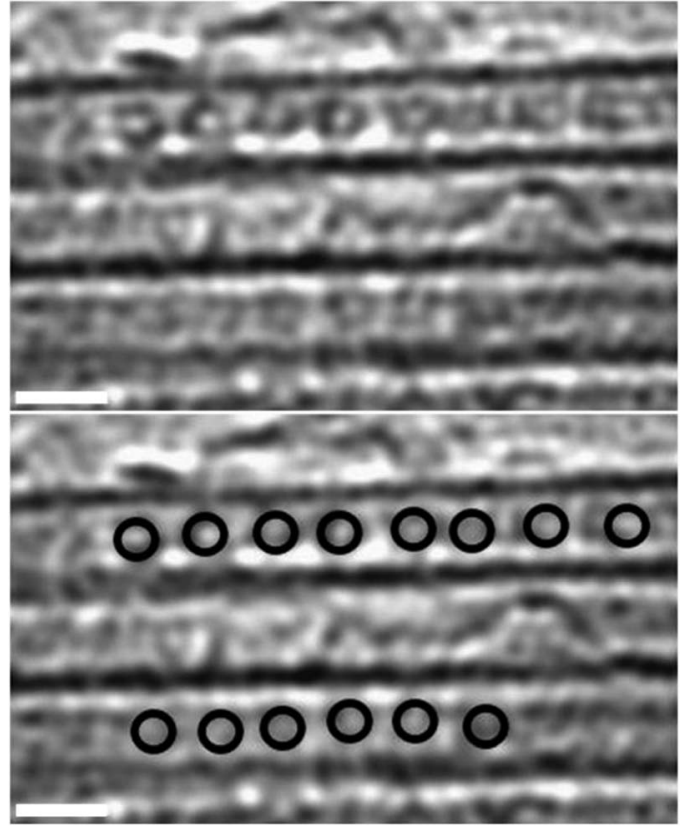

Fig. 4 HRTEM image 6@SWNT. Scale bar 2 nm. In the lower image, the fullerene positions are highlighted by circles.

in Table 2. The values obtained are consistently larger (5-35\%) than those measured experimentally by HRTEM, indicating that upon encapsulation the fullerene molecules are compressed into higher energy conformations within the confines of the nanotube.

HRTEM analysis provides accurate information about the intermolecular spacing of fullerenes in nanotubes, but this information is limited to the local scale. In contrast, XRD provides structural information averaged over a very large number of molecules. Detailed analysis of XRD data can give information on the SWNT-SWNT distances within nanotube bundles and, therefore, on the nanotube diameter, the bundle diameter and the percentage of nanotubes filled with molecules. ${ }^{37}$ As the accurate evaluation of the interfullerene spacing requires sizable periodic structures, the filling ratio is a crucial parameter. Partial filling of an individual nanotube, i.e. a nanotube in which the molecules are not tightly packed, allows the individual molecules to translate along the nanotube quicker than the acquisition time of the XRD, making it impossible to obtain a diffraction peak for fullerenes in partially filled SWNTs. The filling rate for functionalised fullerenes is significantly lower than for pristine $\mathrm{C}_{60}$ (Table 2), presumably due to the presence of the functional groups which hinder entry into the nanotube. However, periodic structures in which functionalised fullerenes are packed regularly inside individual nanotubes were observed by HRTEM, which allowed XRD measurements to be performed for all of the structures in this study.

The XRD patterns for both mono- and bis-functionalised fullerenes in SWNTs are shown in Fig. 5. The interfullerene distances in real space for fullerenes within a SWNT $\left(d_{\text {full }}\right)$ are calculated from the respective maxima in reciprocal space $\left(q_{\max }\right)$ by the formula:

$$
d_{\text {full }}=2 \pi / q_{\text {max }, \text { full }}
$$

and the centre-to-centre distance between SWNTs $\left(d_{100}\right)$ within a large, hexagonally arranged bundle of monodispersed nanotubes with diameter $\left(d_{\text {SWNT }}\right)$ by the formula:

$$
d_{100}=2 \pi / q_{\max , \mathrm{SWNT}} \text { and } d_{\mathrm{SWNT}}=2 d_{100} / \sqrt{3}
$$

where the scattering vector, $q=4 \pi / \lambda \sin \theta, 2 \theta$ is the scattering angle (the angle between the incident and diffracted beams) and $\lambda$ is the wavelength of the X-rays. ${ }^{38}$

Care must be taken when analysing the diffraction patterns as the first peak from a hexagonal arrangement of the nanotubes within a bundle (typically in the range of $q=4-4.5 \mathrm{~nm}^{-1}$ ) is in the same region as peaks due to encapsulated molecular arrays within an individual SWNT with a periodicity of about $1.5 \mathrm{~nm}$. The overlap of these two XRD diffraction peaks can lead to peak a)

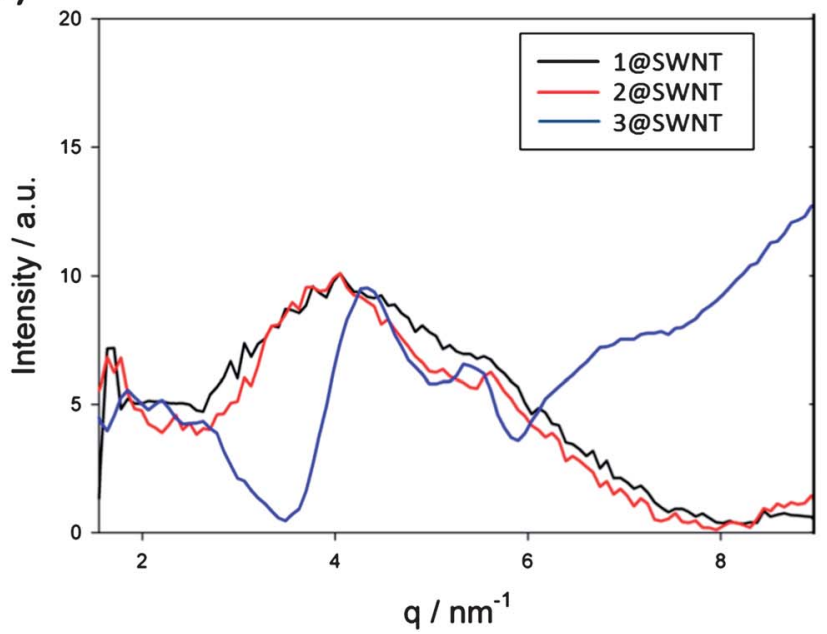

b)

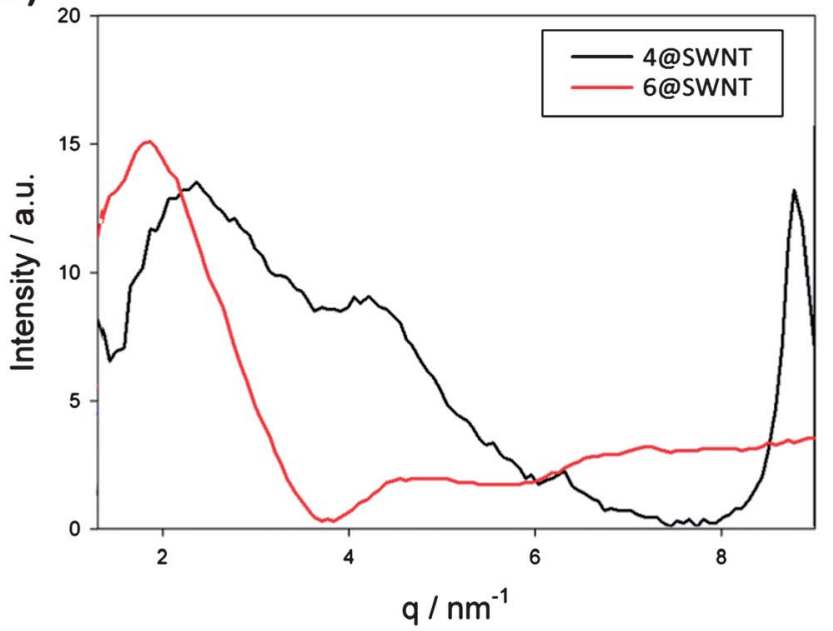

Fig.5 The XRD patterns recorded for structures of (a) mono-functionalised fullerenes 1@SWNT, 2@SWNT and 3@SWNT and (b) bis-functionalised fullerenes4@SWNT and 6@SWNT. 
broadening, thus making it difficult to obtain precise spacings for molecular arrays. For example, in structures 1@SWNT and 2@SWNT, where the fit of the peak maximum gives $d_{\text {full }}=1.55$ and $1.59 \mathrm{~nm}$ respectively, these values have to be taken with caution, as the peak associated with the nanotube bundle and that from the chain of molecules within the nanotubes cannot be separated. Additionally, a very small peak is observed at $q=$ $5.5 \mathrm{~nm}^{-1}$ which can be attributed to a periodic distance of $d_{\text {full }}=$ $\sim 1.1 \mathrm{~nm}$. This corresponds to a head-to-head configuration in which the two fullerene cages are facing each other. In the case of 3@SWNT, the XRD peak at a higher $q$-value gives a distance of $d_{\text {full }}=1.16 \mathrm{~nm}$ for the molecular spacing, which is in excellent agreement with the spacing measured by HRTEM analysis (Table 2 and Fig. 6).

The XRD patterns for bis-functionalised fullerenes 4 and 6 encapsulated in SWNT are shown in Fig. 5b. Due to the lower filling rate of $\mathbf{4}$ and $\mathbf{6}$, the peaks related to the interfullerene spacings are proportionally smaller and can only be distinguished clearly after background subtraction. For the background subtraction of the small-angle X-ray scattering, a powerlaw function with an exponent $n \approx 4$ and a fit constant, $a$, was used:

$$
I_{\text {background }}(q)=a q^{-n}
$$

A value of four for the exponent describes the scattering from objects with a sharp boundary and is known as Porod's law, ${ }^{39}$ whereas a value between three and four arises from a surface fractal or from a rough surface. ${ }^{40}$ In our case, the value of $n \sim 4$ at very small $q$-values indicates agglomerated SWNTs with a bundle diameter larger than $100 \mathrm{~nm}$.

The XRD patterns for structures 4 and $\mathbf{6}$ both show a peak between 4 and $4.5 \mathrm{~nm}^{-1}$ assigned to the hexagonal arrangement of nanotubes within a bundle. The peak observed for 4@SWNT at $q=2.3 \mathrm{~nm}^{-1}$ corresponds to a spacing distance of $d_{\text {full }}=$ $2.7 \mathrm{~nm}$ in real space, which is significantly larger than the value obtained by HRTEM of $1.98 \mathrm{~nm}$. In the XRD pattern recorded for $6 @$ SWNT, the peak position of $q=1.8 \mathrm{~nm}^{-1}$ corresponds to

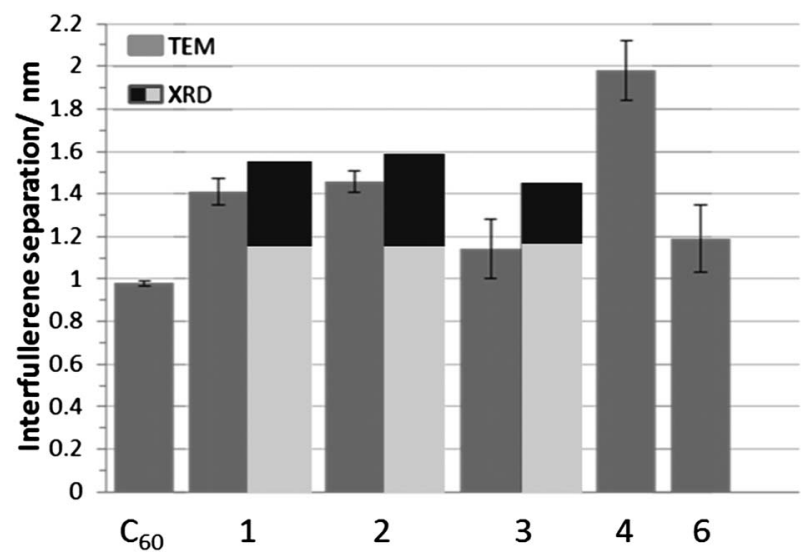

Fig. 6 Spacing between the fullerene cages for the mono-functionalised fullerenes 1, 2, and $\mathbf{3}$ and the bis-functionalised fullerenes $\mathbf{4}$ and $\mathbf{6}$ inside SWNTs as determined from HRTEM and XRD. The bars at the top of each HRTEM column indicate the standard deviation in the measurements. The double-peaked XRD results are indicated with black and light grey (see text). a spacing of $d_{\text {full }}=3.45 \mathrm{~nm}$ between neighbouring fullerenes which is also significantly larger than the value obtained by HRTEM of $1.19 \mathrm{~nm}$ (Table 2).

This could be explained by considering a packing orientation in which adjacent fullerenes are rotated by $90^{\circ}$ with respect to each other (Fig. 7a). In this configuration, the repeating unit would consist of two molecules of $\mathbf{6}$ which explains the larger distances measured by XRD. However, as the origin of this spacing is impossible to verify, the abnormally large intermolecular distance for 6@SWNT is not used for comparison with other systems.

The most intriguing comparison is between the structures formed from the mono- and bis-adducts of $\mathrm{C}_{60}$ and anthracene, 3 and 6. As there are three possible orientations for pairs of fullerene 3 to adopt within a nanotube, HT, HH, and TT, one would expect it to give a less regular structure than 6@SWNT, which can only adopt one orientation (TT). However, experimentally measured interfullerene spacing for the mono-adduct in 3@SWNT $(1.14 \mathrm{~nm})$ is in fact only slightly smaller than the bisadduct, 6@SWNT (1.19), with the standard deviation of the spacing being lower for 3, indicating that 3@SWNT has a more regular structure than $\mathbf{6} @$ SWNT. An explanation for this result is that, upon nanotube filling, a considerable fraction of the bisadduct decomposes to generate the mono-functionalised fullerene via a retro-Diels-Alder reaction (Fig. 7b) which can occur in mild conditions. ${ }^{33}$ This would result in a mixture of mono- and bis-functionalised fullerenes within the nanotube generating a less regular structure. The observation that the mono-adduct, 3, can be formed via decomposition of $\mathbf{6}$ has been reported previously in a Raman spectroscopy study of the two molecules. ${ }^{33}$

The interfullerene spacing in fullerene@SWNT arrays can be considered to be primarily controlled by the physical size of the side-group attached to the fullerene cage. ${ }^{27,28}$ Previously reported crystallographic data obtained from single crystals of $6^{30,41}$ (ESI file $\dagger$ ) can be used to quantify the size of the side-groups and a fullerene-to-fullerene separation (where the cages are separated by the side-group of one of the fullerenes) can be determined as $1.29 \mathrm{~nm}$. Comparison with the experimentally observed separations for 3@SWNT (1.14 nm) and 6@SWNT (1.19 nm) reveals that the observed interfullerene separations are significantly

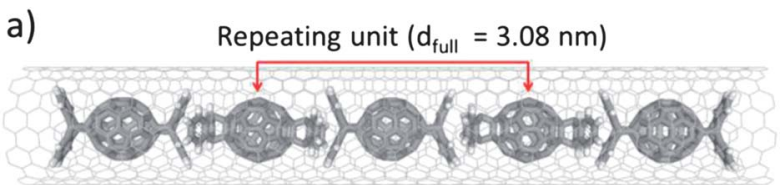

b)

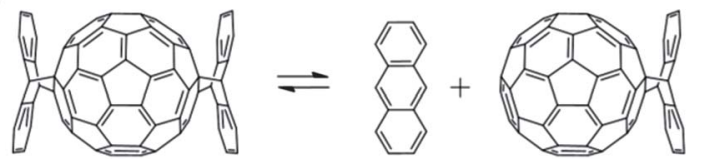

Fig. 7 (a) Schematic diagram depicting the potential packing of 6@SWNT. Due to the $90^{\circ}$ rotation of each fullerene cage with respect to the neighbouring fullerene, the repeating unit is two fullerene cages in length $\left(d_{\text {full }}=3.08 \mathrm{~nm}\right.$ approximated by a space filling model, see ESI file $\dagger$ ). (b) Scheme showing the retro-Diels-Alder reaction of $\mathbf{6}$ to yield $\mathbf{3}$ and anthracene. 
a)

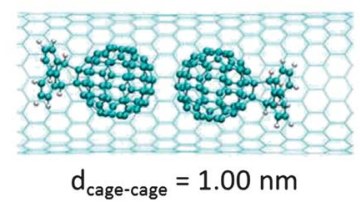

b)

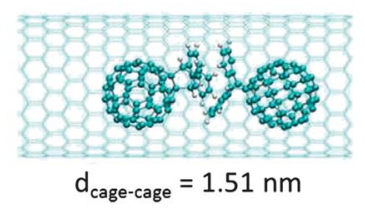

c)

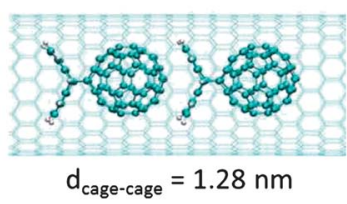

d)

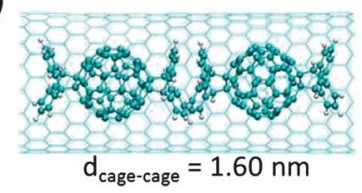

Fig. 8 Lowest energy configurations of 3 inside a $(19,0)$ SWNT $\left(d_{\mathrm{NT}}=\right.$ $1.49 \mathrm{~nm}$ ), for (a) head-to-head, (b) tail-to-tail and (c) head-to-tail orientations and (d) 6@SWNT showing the predicted interfullerene spacings.

shorter in both cases than the distances observed in the $3 \mathrm{D}$ crystal structure of $\mathbf{6}$.

To investigate this, further theoretical simulations were performed for the three different potential orientations of $\mathbf{3}$ and the packing of $\mathbf{6}$ inside a $(19,0)$ SWNT with a diameter of $1.49 \mathrm{~nm}$ matching well the diameters of typical nanotubes in our experimental sample (Fig. 8). The shape of the dihydroanthraceno group enables neat packing of the molecules. In contrast to the previous study in which the planar aromatic tails of the functionalised fullerenes were located between the neighbouring fullerene cage and the nanotube sidewall, ${ }^{28}$ the functional groups of $\mathbf{3}$ and $\mathbf{6}$ are located between the cages providing steric bulk and causing a significant increase in the interfullerene separation. In a head-to-tail orientation, the calculated interfullerene separation is $1.28 \mathrm{~nm}$ which is in excellent agreement with the distance observed in the crystal $(1.29 \mathrm{~nm})$.

Therefore, as the interfullerene spacings predicted by molecular models for3@SWNT and 6@SWNT and the distances observed from the single crystal data of $\mathbf{6}$ both predict larger spacings than those experimentally observed, the nanotube must play an important role in compressing the fullerenes closer together in fullerene@SWNT structures than in 3D crystals. This phenomenon has been observed in a previous study in which smaller than expected interfullerene spacings were reported for functionalised fullerenes encapsulated in SWNTs. This close packing was attributed to the nanotube cavity imparting force upon the fullerenes, forcing them closer together in order to accommodate more fullerene species per unit length of SWNT as a result of the strong van der Waals interactions formed between the fullerene-guest and the host-nanotube. ${ }^{11}$

\section{Experimental}

Arc-discharge produced SWNTs (NanoCarbLab) were used for the experiments. $\mathrm{C}_{60}(99.5 \%)$ was purchased from SES Research. All other reagents and solvents were purchased from Aldrich and were used without further purification. Infrared spectra were measured as either $\mathrm{KBr}$ discs or a solution cell using a Nicolet Avatar 380 FT-IR spectrometer over the range $400-4000 \mathrm{~cm}^{-1}$. ${ }^{1} \mathrm{H}$ - and ${ }^{13} \mathrm{C}$-NMR spectra were obtained using a Bruker DPX 300 spectrometer. Mass spectrometry was carried out using a FAB-LSIMS spectrometer and a MALDI-TOF spectrometer.

The synthesis of $\mathbf{1}$ and $\mathbf{2}$ is based on the work by Mattay et al. ${ }^{29}$

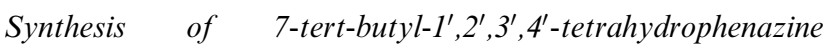
$[1,9]\left(C_{60}-I_{h}\right)[5,6]$-fullerene, $1 . \mathrm{C}_{60}(120 \mathrm{mg}, 0.17 \mathrm{mmol})$ and 1,2-bis(trimethylsiloxy)cyclobutene $(63 \mathrm{mg}, 0.27 \mathrm{mmol})$ were heated under reflux in ortho-dichlorobenzene $(10 \mathrm{~mL})$ under argon for 16 hours. The solvent was removed under reduced pressure to yield a brown solid which was re-dissolved in carbon disulfide $(20 \mathrm{~mL})$, degassed with argon and cooled to $-77^{\circ} \mathrm{C}$. Bromine $(16 \mathrm{mg}, 0.10 \mathrm{mmol})$ was added dropwise and stirred for one hour at $-77{ }^{\circ} \mathrm{C}$ and the solvent was then removed under reduced pressure. A solution of triethylamine trihydrofluoride $(40 \mathrm{mg}, 0.25 \mathrm{mmol})$ in ortho-dichlorobenzene $(10 \mathrm{~mL})$ was degassed for 30 minutes and was added to the reaction mixture, which was then stirred for 1 hour under argon. 4-(tert-Butyl)benzene-1,2-diamine (56 $\mathrm{mg}, 0.34 \mathrm{mmol})$ in glacial acetic acid $(5 \mathrm{~mL})$ was added and the mixture was heated at $60{ }^{\circ} \mathrm{C}$ for three hours. The solvent was removed and purification using silica gel column chromatography with toluene as an eluent yielded the product, $\mathbf{1}$, as a dark brown solid (56 mg, $0.06 \mathrm{mmol}, 35 \%)$. ${ }^{1} \mathrm{H}$ NMR $\left\{400 \mathrm{MHz}, \mathrm{d}_{8^{-}}\right.$ toluene, $300 \mathrm{~K}\} \delta_{\mathrm{H}} 8.44\left(\mathrm{~d},{ }^{4} J=2.4 \mathrm{~Hz}, 1 \mathrm{H}\right.$, aromatic $\left.\mathrm{H}\right), 8.32$ $\left(\mathrm{d},{ }^{3} \mathrm{~J}=8.8 \mathrm{~Hz}, 1 \mathrm{H}\right.$, aromatic $\left.\mathrm{H}\right), 7.63\left(\mathrm{dd},{ }^{3} \mathrm{~J}=8.8 \mathrm{~Hz},{ }^{4} \mathrm{~J}=\right.$ $2.4 \mathrm{~Hz}, 1 \mathrm{H}$, aromatic $\mathrm{H}), 4.74(\mathrm{~m}, 4 \mathrm{H}$, cyclohexane linker $\mathrm{H})$, 1.33 (s, $9 \mathrm{H}, t$-butyl H) ppm. ${ }^{13} \mathrm{C}$ NMR $\left\{100.6 \mathrm{MHz}, \mathrm{d}_{8}\right.$-toluene, $300 \mathrm{~K}\} \delta_{\mathrm{C}} 153.5,153.0,152.8,147.6,146.4,146.1,145.3,144.5$, $142.5,142.0,141.7,140.2,64.8,47.4,47.3,34.8,30.7 \mathrm{ppm}$. MALDI-MS: $932.9 \mathrm{~m} / \mathrm{z}[\mathrm{M}]^{-} \mathrm{C}_{74} \mathrm{H}_{16} \mathrm{~N}_{2}$.

Synthesis of 6,7-bis(bromomethyl)-2,3-di(pyridin-2-yl)quinoxaline, $2 \boldsymbol{a}$. A solution of dimethyl-2,3-di-(2-pyridyl)-quinoxaline $(500 \mathrm{mg}, 1.60 \mathrm{mmol})$, benzoyl peroxide $(36 \mathrm{mg}, 0.15 \mathrm{mmol})$ and $N$-bromosuccinimide $(1.14 \mathrm{~g}, 6.41 \mathrm{mmol})$ in carbon tetrachloride $(50 \mathrm{~mL})$ was heated to $110^{\circ} \mathrm{C}$ for 40 hours under an argon atmosphere. The reaction mixture was then cooled to $0{ }^{\circ} \mathrm{C}$ and filtered by gravity. The filtrate was purified using silica gel column chromatography using chloroform with $1 \%$ methanol as the eluent. The solvent was then removed to leave the product, 2a, as an orange solid ( $372 \mathrm{mg}, 0.79 \mathrm{mmol}, 49 \%)$. ${ }^{1} \mathrm{H}$ NMR $\{400$ MHz, $\mathrm{d}_{6}$-DMSO, $\left.300 \mathrm{~K}\right\} \delta_{\mathrm{H}} 8.42(\mathrm{~m}, 2 \mathrm{H}$, aromatic $\mathrm{H}), 8.26(\mathrm{~m}$, $2 \mathrm{H}$, aromatic $\mathrm{H}), 8.20(\mathrm{~m}, 2 \mathrm{H}$, aromatic $\mathrm{H}), 8.06(\mathrm{~m}, 2 \mathrm{H}$, aromatic $\mathrm{H}), 7.41(\mathrm{~m}, 2 \mathrm{H}$, aromatic $\mathrm{H}), 5.15(\mathrm{~s}, 4 \mathrm{H}, \mathrm{CH} 2) \mathrm{ppm}$. ${ }^{13} \mathrm{C}$ NMR $\left\{100.6 \mathrm{MHz}, \mathrm{d}_{6}\right.$-DMSO, $\left.300 \mathrm{~K}\right\} \delta_{\mathrm{C}} 157.1,153.7$, 148.5, 140.4, 140.0, 137.5, 131.7, 129.7, 129.4, 129.1, 128.7, 124.4, 124.1, 124.0, 79.7, 30.7 ppm. ESI-MS: $470.9 \mathrm{~m} / z \quad[\mathrm{M}]^{+}$ $\mathrm{C}_{20} \mathrm{H}_{14} \mathrm{~N}_{4} \mathrm{Br}_{2}$.

Synthesis of $6^{\prime}, 7^{\prime}, 8^{\prime}, 9^{\prime}$-tetrahydro-2,3-di(pyridin-2-yl)benzoquinoxaline- $[1,9]\left(C_{60}-I_{h}\right)[5,6]$ fullerene, 2. A solution of $\mathrm{C}_{60}$ (100 mg, $0.14 \mathrm{mmol})$, 2a (82 mg, $0.18 \mathrm{mmol})$, 18-crown-6 $(300 \mathrm{mg}, 1.14 \mathrm{mmol})$ and potassium iodide $(50 \mathrm{mg}, 0.30 \mathrm{mmol})$ in dry toluene $(50 \mathrm{~mL})$ was refluxed for 16 hours. The solvent was removed under reduced pressure and purification using silica gel column chromatography with toluene and ethyl acetate $(3: 1)$ as the eluant yielded the product, 2 , as a brown solid $(35 \mathrm{mg}, 0.034$ mmol, 24\%). ${ }^{1} \mathrm{H}$ NMR $\left\{300 \mathrm{MHz}, \mathrm{CDCl}_{3}, 298 \mathrm{~K}\right\} \delta_{\mathrm{H}} 8.49(\mathrm{~s}, 2 \mathrm{H}$, aromatic $\mathrm{H}), 8.32\left(\mathrm{~d},{ }^{3} \mathrm{~J}=4.2 \mathrm{~Hz}, 2 \mathrm{H}\right.$, aromatic $\left.\mathrm{H}\right), 8.13\left(\mathrm{~d},{ }^{3} \mathrm{~J}=\right.$ $4.2 \mathrm{~Hz}, 2 \mathrm{H}$, aromatic $\mathrm{H}), 7.87\left(\mathrm{t},{ }^{3} J=7.8 \mathrm{~Hz}\right.$, aromatic $\left.\mathrm{H}\right), 7.26$ $\left(\mathrm{t},{ }^{3} \mathrm{~J}=7.8 \mathrm{~Hz}, 2 \mathrm{H}\right.$, aromatic $\left.\mathrm{H}\right), 5.17\left(\mathrm{~d},{ }^{2} \mathrm{~J}=13.8 \mathrm{~Hz}\right.$, cyclohexane linker $\mathrm{H}), 4.77\left(\mathrm{~d},{ }^{2} J=13.8 \mathrm{~Hz}\right.$, cyclohexane linker $\left.\mathrm{H}\right)$ ppm. ${ }^{13} \mathrm{C}$ NMR $\left\{75 \mathrm{MHz}, \mathrm{CDCl}_{3}, 298 \mathrm{~K}\right\} \delta_{\mathrm{C}} 148.1,145.6,136.2$, 128.2, 124.2, 122.8, 45.4, $30.2 \mathrm{ppm}$. MALDI-MS: $1030.50 \mathrm{~m} / \mathrm{z}$ $[\mathrm{M}]^{-} \mathrm{C}_{80} \mathrm{H}_{14} \mathrm{~N}_{4}$. 
The synthesis of $\mathbf{3}$ and $\mathbf{6}$ is based on the work of Kräutler and co-workers. ${ }^{30-33}$ The bis-functionalised fullerene 4 was prepared utilizing a multi-step synthesis outlined by Qian and Rubin. ${ }^{34}$ Details for the synthesis of $\mathbf{5}$ are reported in the work of Milic and Prato. ${ }^{35}$

\section{Filling of single-walled carbon nanotubes}

SWNTs (diameter of $1.39 \mathrm{~nm}$ ) were used for the experiments. The purified nanotubes (supplied $80 \%$ pure) were annealed in air at $540{ }^{\circ} \mathrm{C}$ for 20 minutes to remove amorphous carbon and to open their termini; a weight loss of approximately $20 \%$ was observed. The sample was then stored at $400{ }^{\circ} \mathrm{C}$ to prevent water from condensing in the internal cavities of the nanotubes.

A three-fold excess of fullerene was dispersed in $\mathrm{CHCl}_{3}(1 \mathrm{~mL})$ using an ultrasonic bath to form a super-saturated solution which was added dropwise to the freshly annealed nanotubes in an agate mortar allowing each drop to evaporate before adding a further drop. The resultant black solid was allowed to dry thoroughly in air and then ground using a pestle and mortar for 5 minutes before being washed with carbon disulfide $(20 \mathrm{~mL})$ to remove any nonencapsulated fullerenes and then washed with methanol $(20 \mathrm{~mL})$. Approximately $0.5 \mathrm{mg}$ of each sample was dispersed in methanol $(2 \mathrm{~mL})$ using an ultrasonic bath and the resultant suspensions were drop cast onto amorphous carbon coated copper TEM grids.

\section{TEM imaging}

HRTEM analysis was performed on a JEOL-2100F TEM microscope with a resolution limit of $0.2 \mathrm{~nm}$. The imaging conditions were carefully tuned by lowering the accelerating voltage of the microscope to $100 \mathrm{kV}$ and reducing the beam current density to a minimum. Under these conditions the positions of fullerene cages in nanotubes could be clearly observed, which were used for determining intermolecular separations. The filling rates were determined by taking micrographs of $100 \mathrm{~nm}^{2}$ areas from different regions of the specimen, estimating the proportion of filled nanotubes for each area and by averaging the filling factors over several areas. ${ }^{11}$

\section{XRD measurements}

XRD measurements were performed in three or more different positions for each sample with X-rays from a rotating anode generator equipped with a pinhole camera $(\mathrm{Cu} \mathrm{K} \alpha$ radiation with a wavelength of $\lambda=0.1542 \mathrm{~nm}$, monochromatised and collimated with crossed Göbel mirrors from Bruker AXS, Karlsruhe). All X-ray diffraction patterns were radially averaged and corrected for background scattering to obtain the scattering intensity $I(q)$, where $q=(4 \pi / \lambda) \sin \theta$, the magnitude of the scattering vector $q=|\vec{q}|$, and $2 \theta$ is the angle between the incident and diffracted beams.

\section{Molecular models calculations}

The theoretical calculations were carried out by simulating the nanotubes with the Brenner potential. ${ }^{42}$ The functionalised fullerenes were described by a general potential for organics. ${ }^{43}$ The van der Waals interactions between nanotubes and molecules were also described by a nonbonding potential that was calibrated to reproduce the interactions in graphite and in benzene dimers ${ }^{44}$ using the potential energy function:

$$
V(r)=\varepsilon_{\mathrm{V}}\left[1840 \exp \left(-12 \frac{r}{r_{\mathrm{v}}}\right)-2.25\left(\frac{r}{r_{\mathrm{v}}}\right)^{6}\right]
$$

where $r$ is the inter-atomic distance, $r_{\mathrm{v}}=1.960 \AA$ and $\varepsilon_{\mathrm{v}}=0.056$ $\mathrm{kcal} \mathrm{mol}^{-1}$.

All of the calculations were performed using a modified version of the TINKER molecular mechanics dynamics software package ${ }^{4-46}$ with enforced periodic boundary conditions. The nanotube unit cell was extended to $4 \mathrm{~nm}$ and the cut-off of the potential energy functions was set to $1.2 \mathrm{~nm}$. In order to fully explore the potential energy hypersurface, a computer program originally developed to investigate fullerenes in nanotubes employing the Rigid Molecule Random Explorer (RMRE) algorithm was used. This randomly arranges the molecules within the nanotube and rigidly minimizes the energy for a large number of potential configurations. ${ }^{47,48}$ Initially at least 1000 cycles of RMRE were used to generate the starting points for the functionalised fullerene structures, and then full geometry minimisation of the fullerene@nanotube was performed. The geometries were optimised to a root mean square deviation of the energy gradient below $0.01 \mathrm{kcal} \mathrm{mol}^{-1} \AA^{-1}$.

\section{Conclusions}

This study presents the synthesis of six functionalised fullerenes designed to facilitate the controlled ordering of molecular chains within nanotube cavities. The fullerenes were inserted into SWNTs using a solution-based filling method under mild conditions. The resultant structures were characterised using HRTEM and XRD, demonstrating that the spacing between neighbouring fullerene cages can be effectively controlled by using steric bulk of functional groups attached to the fullerenes. The orientation of molecules within SWNTs appears to be crucial for controlling the intermolecular distance. The problem of random orientations was successfully addressed by introducing bipodal functionalised fullerenes bearing sterically bulky, conformationally rigid groups on opposite sides of the $\mathrm{C}_{60}$ cage. Our methodology offers a powerful approach for constructing 1D molecular arrays which can be potentially exploited in molecular spintronics or quantum information processing devices.

\section{Acknowledgements}

This work was financially supported by FWF project I83-N20 (ESF IMPrESS), the Royal Society, the European Research Council (ERC), "Fundação para a Ciência ea Tecnologia" through the program Ciência 2008, the project SeARCH (Services and Advanced Research Computing with HTC/HPC clusters) and Nottingham Nanoscience and Nanotechnology Centre (NNNC).

\section{Notes and references}

1 A. N. Khlobystov, D. A. Britz and G. A. D. Briggs, Acc. Chem. Res., 2005, 38, 901. 
2 B. W. Smith, M. Monthioux and D. E. Luzzi, Chem. Phys. Lett., 1999, 315,31 .

3 A. N. Khlobystov, K. Porfyrakis, M. Kanai, D. A. Britz, A. Ardavan, H. Shinohara, T. J. S. Dennis and G. A. D. Briggs, Angew. Chem., Int. Ed., 2004, 43, 1386.

4 D. A. Britz, A. N. Khlobystov, K. Porfyrakis, A. Ardavan and G. A. D. Briggs, Chem. Commun., 2005, 37.

5 A. Chuvilin, E. Bichoutskaia, M. C. Gimenez-Lopez, T. W. Chamberlain, G. A. Rance, N. Kuganathan, J. Biskupek, U. Kaiser and A. N. Khlobystov, Nat. Mater., 2011, 10, 687.

6 H. Ulbricht and T. Hertel, J. Phys. Chem. B, 2003, 107, 14185.

7 M. del Carmen Gimenez-Lopez, A. Chuvilin, U. Kaiser and A. N. Khlobystov, Chem. Commun., 2011, 47, 2116.

8 M. Koshino, N. Solin, T. Tanaka, H. Isobe and E. Nakamura, Nat. Nanotechnol., 2008, 3, 595.

9 N. Solin, M. Koshino, T. Tanaka, S. Takenaga, H. Kataura, H. Isobe and E. Nakamura, Chem. Lett., 2007, 36, 1208.

10 J. Fan, T. W. Chamberlain, Y. Wang, S. Yang, A. J. Blake, M. Schröder and A. N. Khlobystov, Chem. Commun., 2011, 47, 5696.

$11 \mathrm{~T}$. W. Chamberlain, N. R. Champness, M. Schröder and A. N. Khlobystov, Chem.-Eur. J., 2011, 17, 668.

12 E. Nakamura, M. Koshino, T. Saito, Y. Niimi, K. Suenaga and Y. Matsuo, J. Am. Chem. Soc., 2011, 133, 14151.

13 S. Campestrini, C. Corvaja, M. De Nardi, C. Ducati, L. Franco, M. Maggini, M. Meneghetti, E. Menna and G. Ruaro, Small, 2008, 4, 350 .

14 F. Simon, H. Kuzmany, J. Bernardi, F. Hauke and A. Hirsch, Carbon, 2006, 44, 1958.

15 F. Simon, H. Kuzmany, B. Náfrádi, T. Fehér, L. Forró, F. Fülop, A. Jánossy, L. Korecz, A. Rockenbauer and F. Hauke, et al., Phys. Rev. Lett., 2006, 97, 136801.

16 B. Corzilius, A. Gembus, N. Weiden, K.-P. Dinse and K. Hata, Phys. Status Solidi B, 2006, 243, 3273.

17 F. Simon, H. Kuzmany, H. Rauf, T. Pichler, J. Bernardi, H. Peterlik, L. Korecz, F. Fülop and A. Jánossy, Chem. Phys. Lett., 2004, 383 , 362 .

18 J. Lee, H. Kim, S.-J. Kahng, G. Kim, Y.-W. Son, J. Ihm, H. Kato, Z. W. Wang, T. Okazaki and H. Shinohara, et al., Nature, 2002, 415, 1005 .

19 D. J. Hornbaker, S.-J. Kahng, S. Misra, B. W. Smith, A. T. Johnson, E. J. Mele, D. E. Luzzi and A. Yazdani, Science, 2002, 295, 828.

20 L.-J. Li, A. N. Khlobystov, J. G. Wiltshire, G. A. D. Briggs and R. J. Nicholas, Nat. Mater., 2005, 4, 481.

21 E. V. Basiuk, E. V. Rybak-Akimova, V. A. Basiuk, D. AcostaNajarro and J. M. Saniger, Nano Lett., 2002, 2, 1249.

22 S. C. Benjamin, A. Ardavan, G. A. D. Briggs, D. A. Britz, D. Gunlycke, J. Jefferson, M. A. G. Jones, D. F. Leigh, B. W. Lovett, A. N. Khlobystov, S. A. Lyon, J. J. L. Morton, K. Porfyrakis, M. R. Sambrook and A. M. Tyryshkin, J. Phys. Condens. Matter, 2006, 18, S867.
23 A. N. Khlobystov, in Chemistry of Carbon Nanotubes, ed. V. A. Basiuk and E. V. Basiuk, American Scientific Publishers, California, 2007.

24 T. Lu, E. M. Goldfeld and S. K. Gray, J. Phys. Chem. C, 2008, 112, 2654.

25 M. D. Halls and H. B. Schlegel, J. Phys. Chem. B, 2002, 106, 1921.

26 M. Zaka, J. H. Warner, Y. Ito, J. J. L. Morton, M. H. Rummeli, T. Pichler, A. Ardavan, H. Shinohara and G. A. D. Briggs, Phys. Rev. B: Condens. Matter Mater. Phys., 2010, 81, 075424.

27 T. W. Chamberlain, A. Camenisch, N. R. Champness, G. A. D. Briggs, S. C. Benjamin, A. Ardavan and A. N. Khlobystov, J. Am. Chem. Soc., 2007, 129, 8609.

28 T. W. Chamberlain, R. Pfeiffer, H. Peterlik, H. Kuzmany, F. Zerbetto, M. Melle-Franco, L. Staddon, N. R. Champness, G. A. D. Briggs and A. N. Khlobystov, Small, 2008, 4, 2262.

29 J. Mattay, G. Torres-Garcia, J. Averdung, C. Wolf, I. Schlachter, H. Luftmann, C. Siedschlag, P. Luger and M. Ramm, J. Phys. Chem. Solids, 1997, 58, 1929.

30 B. Kräutler, T. Müller, J. Maynollo, K. Gruber, C. Kratky, P. Ochsenbein, D. Schwarzenbach and H.-B. Burgi, Angew. Chem., Int. Ed., 1996, 35, 1204.

31 A. Duarte-Ruiz, T. Müller, K. Wurst and B. Kräutler, Tetrahedron, $2001, \mathbf{5 7}, 3709$.

32 B. Kräutler, T. Müller and A. Duarte-Ruiz, Chem.-Eur. J., 2001, 7, 3223.

33 R. Pfeiffer, C. Kramberger, H. Peterlik, H. Kuzmany, B. Kräutler, M. Melle-Franco and F. Zerbetto, Phys. Status Solidi B, 2009, 246, 2794.

34 W. Qian and Y. Rubin, Angew. Chem., Int. Ed., 1999, 38, 2356.

35 D. Milić and M. Prato, Eur. J. Org. Chem., 2010, 476.

36 D. A. Britz and A. N. Khlobystov, Chem. Soc. Rev., 2006, 35, 637.

37 J. Cambedouzou, V. Pichot, S. Rols, P. Launois, P. Petit, R. Klement, H. Kataura and R. Almairac, Eur. Phys. J. B, 2004, 42, 31.

38 S. Rols, R. Almairac, L. Henrard, E. Anglaret and J. L. Sauvajol, Eur. Phys. J. B, 1999, 10, 263

39 G. Porod, Kolloid Z., 1951, 124, 83.

40 A. Emmerling, J. Fricke and J. Non-Cryst, Solids, 1992, 145, 113.

41 A. Duarte-Ruiz, K. Wurst and B. Kräutler, Helv. Chim. Acta, 2008, 91, 1401.

42 D. W. Brenner, Phys. Rev. B: Condens. Matter Mater. Phys., 1990, 42, 9458.

43 N. L. Allinger, Y. H. Yuh and J.-H. Lii, J. Am. Chem. Soc., 1989, 23, 8551 .

44 J. W. Ponder and F. J. Richards, J. Comput. Chem., 1987, 8, 1016.

45 C. J. Kundrot, W. Ponder and F. J. Richards, J. Comput. Chem., 1991, 12, 402.

46 M. J. Dudek and J. W. Ponder, J. Comput. Chem., 1995, 16, 791.

47 R. Pfeiffer, H. Kuzmany, T. Pichler, H. Kataura, Y. Achiba, M. Melle-Franco and F. Zerbetto, Phys. Rev. B: Condens. Matter Mater. Phys., 2004, 69, 035404.

48 M. Melle-Franco, H. Kuzmany and F. Zerbetto, J. Phys. Chem. B, 2003, 107, 6986 